\title{
Dynamics Modeling and Analysis of Oligo DNA Microarray Spotting
}

\author{
Mingjun Zhang \\ Agilent Technologies, California, USA \\ Email: mingjunzhang@ieee.org
}

\author{
$\mathrm{Ou} \mathrm{Ma}$ \\ New Mexico State University, New Mexico, USA \\ Email: oma@nmsu.edu
}

\begin{abstract}
Oligo DNA microarrays are fabricated through in-situ chemical synthesis. Contact and fluid dynamics all contribute to this process. To produce high quality oligo DNA microarrays, it is important to understand dynamics of the process well. Much work has been done in understanding the chemistry principles. However, few studies have been conducted from mechanics point of view. This paper proposes a contact dynamic model of oligo DNA microarray spotting process. Simulation results using the proposed dynamic model can reasonably well explain dynamics of the microarray spotting process.
\end{abstract}

\section{INTRODUCTION}

DNA microarray is a widely accepted tool for high throughput genomics research. There are in general two kinds of DNA microarrays. One is called complimentary DNA (cDNA) microarrays. The other is oligonucleotide DNA (oDNA or oligo DNA) microarrays. Because of its nice specificity and sensitivity, the oligo DNA microarray is emerging as a dominant platform for genomics research. The oligo DNA microarrays are usually fabricated through in-situ chemical synthesis using solid phase phosphoramidite chemistry.

Two major methods currently being used to fabricate oligo DNA microarrays are photo-directed lithography technique extended from semiconductor manufacturing and the inkjet printing technology [8]. The oligo DNA microarrays are usually built layers by layers. Each layer adds one nucleotide onto an existing single stranded DNA tethered to a substrate surface. The exact building address is controlled by controlling the reaction site. This is because DNA insitu chemical synthesis can take place only by elongation of the growing strand in the $5^{\prime}$-to- $3^{\prime}$ direction through the addition of successive nucleotides to the $3^{\prime}$ end. After the first base is printed, the chemical group that protects the $5^{\prime}$ hydroxyl group on the nucleotide is removed and oxidized to activate and react with the $3^{\prime}$ group on the next nucleotide. In between different steps, the excess chemical reagents are washed away. After the last base in the oligo chain is printed, the microarrays undergo a final de-protection step, before being moved to scribing, dicing and quality control testing.

The above oligo DNA microarray fabrication is a sophisticated chemical reaction and micro-scale manufacturing process. The underlying atomic level chemical bonding affects molecular level interactions, which in turn affects microarray synthesis for each spot at micro-scale. Even though it is well-known for how the single-stranded DNA is built chemically, it is still an open issue for how dynamics plays a role in this sophisticated process. It has been observed in practice that the synthesis rate is sensitive to density of the spotted nucleotide solution and environmental conditions, and is mechanically fragile to washing conditions. However, these observations have not been well understood.

Dynamic modeling and analysis would be helpful to answer these questions. Unfortunately, the microarray fabrication process is in nano-scale, which lacks of theoretical understandings. The modeling techniques capable to model nano-scale mechanics of such a process has not been well established in the open literature. Paper [6] reported some interesting results on modeling nano-particle dynamics. The results are primarily for AFM based nano-particle manipulation. Paper [1] discusses some fundamental physical phenomena in micro-scale manipulation. For the oligo DNA microarray fabrication, contact dynamics of multiple nanoparticles needs to be considered. The challenge is not only on building and validating a mathematical model that reflects the nano-scale molecular dynamics and spotting mechanics, but also on proposing a model that is computational efficient for implementation.

This paper proposes a dynamic model of the droplet contact dynamics for the nucleotide spotting process. The nucleotides are modeled as cylindrical objects. Contact forces, electrostatic force, viscous force and van der Waals forces have all been considered. One challenging question is how to verify the dynamic model. The best way is probably through experimental studies. Unfortunately, real time monitoring of the DNA in-situ synthesis is not only very difficult but also impossible at present time [4]. In this paper, simulation results at nano-scale will be presented and discussed with chemical principles.

This paper is organized as follows. In Section II, the nucleotide spotting process is formulated as a multi-body contact dynamics problem. Newtonian particle mechanics is then applied. Simulation results for the formulation are presented in Section III. In Section IV, discussions and conclusive remarks are given. 


\section{PROBlem FORMULATION}

For oligo DNA microarray fabrication, the phosphate group of the nucleotide in the first droplet reacts with a hydroxyl-group of the linker molecule coated on a substrate surface. After washing away excess nucleotides, the chemical capping material of the top nucleotide is removed, and another droplet is spotted onto the exact address. This process repeats multiple times to build single oligonucleotide sequence. During the fabrication, a nano-liter or even picoliter droplet containing approximately $6 \times 10^{12}$ same single nucleotide bases with chemically modified end of capping material is spotted onto exact address of a substrate surface. The spotted DNA feature usually runs from 50 to $150 \mu \mathrm{m}$. Each nucleotide is about $1.0 \mathrm{~nm}$ in diameter and $0.34 \mathrm{~nm}$ in length. This means that there are thousands of single stranded DNA at each printed microarray spot.

One critical step for the above chemical synthesis is the phosphate and hydroxyl group binding process (called coupling), which may take place through the Brownian motion. However, the binding process is too slow, if it is only dependent on the Brownian motion. To speed up the process, the $3^{\prime}$ single stranded DNA is usually modified by a chemical group, which is one unit positively charged. The nucleotide is normally one unit negatively charged. The electrical charge interaction force between the positively and negatively charged molecules, along with the van der Waals force, become the dominant attraction forces to bring the nucleotides together. In addition, viscous force also contributes to this in-situ synthesis process.

One possible formulation of the above dynamics would be single nucleotide level dynamics with all-atom molecular dynamics. Unfortunately, the number of variables introduced by such an approach are too large to be dealt with in the Newtonian realm of physics and mathematical formulation. Another possibility is kilo-base pair level modeling by macro-scopic models using Monte Carlo, Brownian, and Langevin dynamics [5]. The concern is that this may not match the oligo DNA microarray spotting process well, where nucleotides are ejected by droplet. The third possibility is micro-fluidic dynamic modeling, whose scale seems too large to be understood for necessary details.

Our interest in this paper is on contact dynamics of the nucleotide droplets to the growing single stranded DNA molecules. We will formulate the problem using contact dynamics of the Newtonian mechanics. Molecular level interactions and contact impact forces will be considered. It is our hope to make the model complex enough to have a good fidelity of the real dynamics of the spotting process, and simple enough to have a practical understanding of the process.

A nucleotide usually consists of a base (one of the following four chemicals: adenine, thymine, guanine, and cytosine) pluses a molecule of sugar and one of phosphoric acid. It is the variations in bases that makes the four different nucleotides representing by $A, T, G$ and $C$. Each nucleotide is in general nicely compacted and bonded. It is close to a cylinder shape with an approximate diameter of $1.0 \mathrm{~nm}$ and a length of $0.34 \mathrm{~nm}$. Molecular masses of the four nucleotides A, T, G, C are 329.21 a.m.u., 320.19 a.m.u., 345.21 a.m.u., and 305.18 a.m.u., respectively.

In general, geometric differences of the above four nucleotides are not significant. Since we are formulating the nucleotides as mechanical objects, it is reasonable to assume that they all have similar mechanical properties. Some additional assumptions are:

- The nuclear and gravitational interactions are neglected. Van der Waals forces will be considered instead.

- Quantum mechanics will not be applied. Quantum mechanics adds heavy burdens on the computation. However, the final results may not be affected much compared to classical mechanics methods for the problem of interest.

- Each nucleotide is assumed to be subjected to contact forces, electrostatic forces, viscous forces and van der Waals forces from its neighboring nucleotides. The contact forces can be regarded as forces contributed from impact, elasticity, surface damping, and geometric constraints. Repulsive force between two atoms is attributed to the contact force. The underline fundamental principle for this assumption is that atoms have a radius and cannot be overlapped. Sine the repulsive force drops dramatically as the distance between two atoms increases, but conversely becomes very large at short distance.

- Different from problems dealt with in molecular dynamics, the nucleotides are assumed to be ejected with a known initial velocity and a possible lateral offset in position.

- The viscous force is modeled as the main interactions with environmental media.

Based on the above assumptions, the dynamic model of the oligonucleotide DNA microarray spotting process is proposed in Fig. 1. In the model, each nucleotide is approximated by a three-dimensional cylindrical object. Multinucleotides form a single stranded DNA through covalent coupling. Each time when a new nucleotide is added on, a droplet containing multiple millions of the same nucleotides is spotted on top of the strand. A free-body diagram of three neighboring nucleotides is shown in Fig. 2.

By the Newtonian mechanics, dynamics of the $i$-th nucleotide of the single stranded DNA can be represented by the following equations.

$$
\begin{gathered}
\left(\mathbf{f}_{\mathbf{v}}\right)_{\mathbf{i}}+\left(\mathbf{f}_{\mathbf{c}}\right)_{\mathbf{i}}+\left(\mathbf{f}_{\mathbf{e}}\right)_{\mathbf{i}}+\left(\mathbf{f}_{\mathbf{v w}}\right)_{\mathbf{i}}-\left(\mathbf{f}_{\mathbf{c}}\right)_{\mathbf{i}+\mathbf{1}} \\
-\left(\mathbf{f}_{\mathbf{v w}}\right)_{\mathbf{i}+\mathbf{1}}=m_{i} \ddot{\mathbf{p}}_{\mathbf{i}} \\
\left(\tau_{\mathbf{v}}\right)_{\mathbf{i}}+\mathbf{r}_{\mathbf{i}} \times\left(\mathbf{f}_{\mathbf{c}}\right)_{\mathbf{i}}-\mathbf{r}_{\mathbf{i}+\mathbf{1}} \times\left(\mathbf{f}_{\mathbf{c}}\right)_{\mathbf{i}+\mathbf{1}}=\mathbf{I}_{\mathbf{i}} \dot{\omega}_{\mathbf{i}} \\
+\omega_{\mathbf{i}} \times\left(\omega_{\mathbf{i}} \times \mathbf{I}_{\mathbf{i}} \omega_{\mathbf{i}}\right)
\end{gathered}
$$

where $i=1,2, \cdots, n$, and $n$ is the total number of nucleotides in a nucleotide strand.

In equation $(1)$, vector $\left(\mathbf{f}_{\mathbf{c}}\right)_{\mathbf{i}}$ represents the contact force exerted on the $i$-th nucleotide by the $(i-1)$-th nucleotide. 


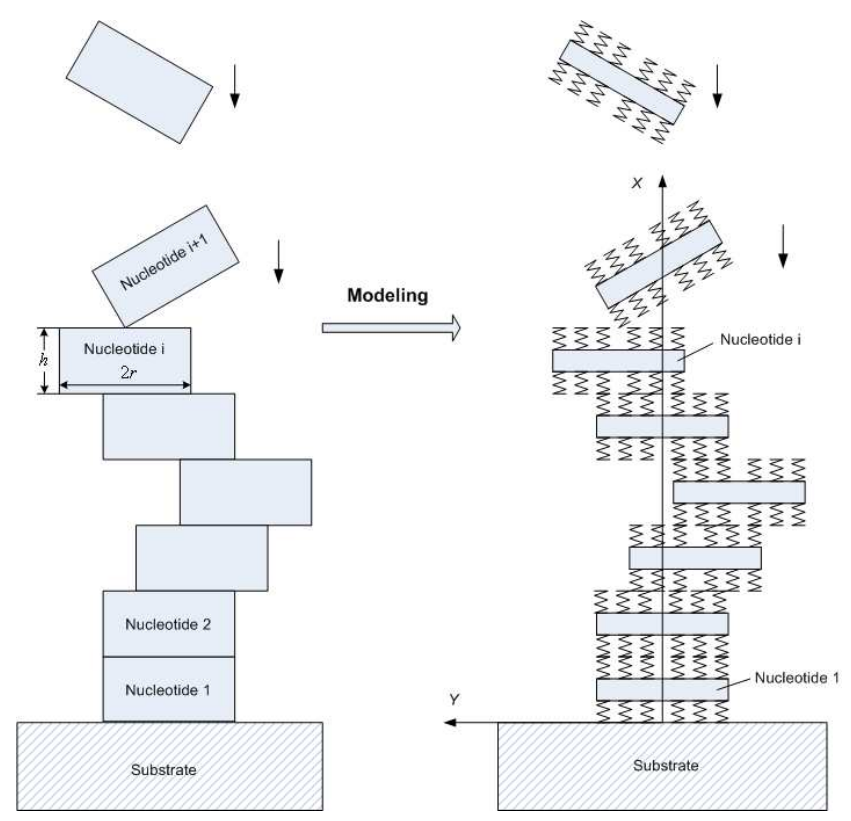

Fig. 1. Dynamic Model of the Nucleotide Spotting Process
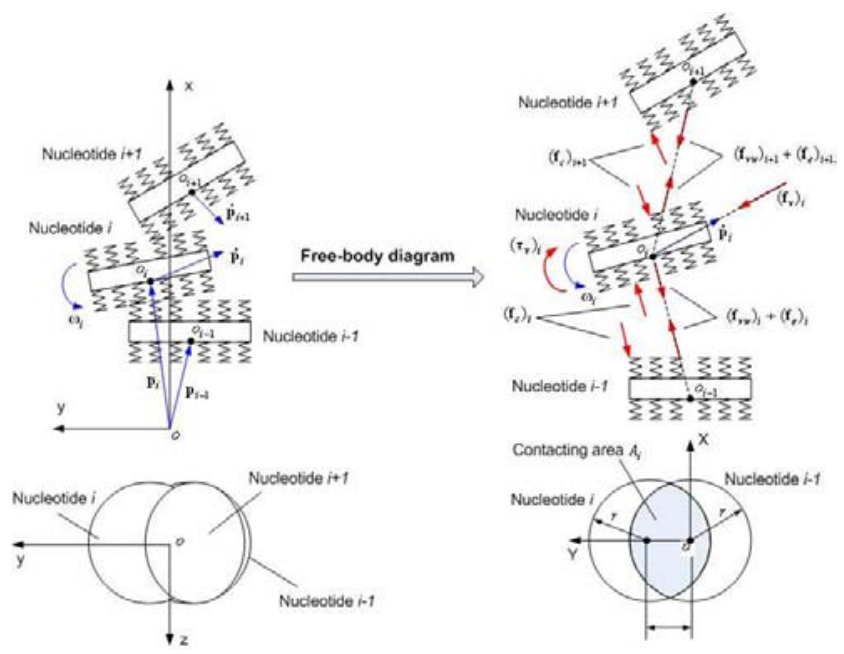

Fig. 2. A Free-body Diagram of Three Neighboring Nucleotides

Vectors $\left(\mathbf{f}_{\mathbf{e}}\right)_{\mathbf{i}}$ and $\left(\mathbf{f}_{\mathbf{v w}}\right)_{\mathbf{i}}$ stand for the electrostatic and the van der Waals forces exerted on the $i$-th nucleotide by all the other nucleotides in the strand. Vectors $\left(\mathbf{f}_{\mathbf{v}}\right)_{\mathbf{i}}$ and $\left(\tau_{\mathbf{v}}\right)_{\mathbf{i}}$ represent the viscous force and torque exerted on the $i$ th nucleotide through environmental fluid. Vectors $\ddot{\mathbf{p}}_{\mathbf{i}}$ and $\omega_{\mathbf{i}}$ represent the linear acceleration and angular velocity of the $i$-th nucleotide. The $m_{i}$ represents the mass, and $\mathbf{I}_{\mathbf{i}}$ represents a $3 \times 3$ second order inertial matrix of the nucleotide. Vector $\mathbf{r}_{\mathbf{i}}$ points to the contact location from the mass center of the $i$-th nucleotide. It is assumed that contact forces are applied through center of the contact area between the two nucleotides. When the single stranded DNA is at a static equilibrium, all forces and torques should be in balance.

The force $\left(\mathbf{f}_{\mathbf{c}}\right)_{\mathbf{i}}$ is defined as $-\left(f_{c}\right)_{i} \mathbf{n}_{\mathbf{i}}$, where $\mathbf{n}_{\mathbf{i}}$ is a unit vector along the effective surface normal direction at the contact point. The Hertz contact model is used in the formulation, which can be expressed in terms of the surface deformation $\delta_{i}$ and its time derivative $\dot{\delta}_{i}$ between every two contacting nucleotides as shown in equation (2).

$$
\left(f_{c}\right)_{i}= \begin{cases}k_{c}\left|\delta_{i}\right|^{\frac{3}{2}}-c_{c} \dot{\delta}_{i}, & \text { if } \delta_{i} \leq 0 \text { (in contact) } \\ 0, & \text { otherwise (no contact) }\end{cases}
$$

where $k_{c}$ and $c_{c}$ are linear contact stiffness and contact damping coefficients. These two parameters reflect the mechanical properties of the nucleotides. The $\delta_{i}$ is defined as the closest distance between the two neighboring nucleotides if they are not in contact, and the collision indentation if they are in contact.

\section{A. Viscous Force}

A nucleotide is subject to viscous drag when it moves inside a liquid droplet. Assume that the relative flow motion near the surface of a nucleotide is laminar and the geometry of the nucleotide can be approximated using a sphere. The viscus force can be regarded as proportional to the relative velocity of the nucleotide in the liquid, namely,

$$
\begin{aligned}
& \left(\mathbf{f}_{\mathbf{v}}\right)_{\mathbf{i}}=-c_{f} \dot{\mathbf{p}}_{\mathbf{i}} \\
& \left(\tau_{\mathbf{v}}\right)_{\mathbf{i}}=-c_{\tau} \omega_{\mathbf{i}}
\end{aligned}
$$

where $c_{f}$ and $c_{\tau}$ are the translational and rotational viscous coefficients. Based on the Stokes law, $c_{f}=6 \pi r \mu$ where $r$ is the radius of the sphere and $\mu$ is the viscosity of the fluid.

\section{B. Electrostatic forces}

In this formulation, only formal charges of nucleotide will be considered. Formal charges show whether chemical groups are ionized. According to the Coulomb's law [2], the electrostatic force can be calculated based on the following equations

$$
\left(f_{e}\right)_{i j}=\frac{q_{i} q_{j}}{4 \pi \varepsilon_{0} \varepsilon_{r} \delta_{i j}^{2}}
$$

where $q_{i}$ and $q_{j}$ are magnitudes of the charges of two nucleotides; $\delta_{i j}$ is their separation; $\varepsilon_{0}$ is the permittivity of free space; and $\varepsilon_{r}$ is the relative dielectric constant of the medium in which the charges are placed. The line of action of the electrostatic force is assumed to be along the centroids of the two involved nucleotides in our formulation.

\section{Van der Waals Force}

The van der Waals force is a short range force caused by instantaneous dipole. The instantaneous dipole is usually generated by the fluctuation of electron cloud surrounding the nucleus of electrically neutral atoms. The van der Waals force decays rapidly to zero away from a surface. It is comparatively larger in air than in a media, and it is proportional to the Hamaker constant. The Hamaker constant is one of the leading parameters to accurately estimate the van der Waals forces. The Hamaker constant mainly depends on the materials of the objects in interaction and the medium that separates them. 
In [7], van der Waals energy expressions have been given for two shells, two parallel walls, a shell and a sphere for same media. No expression for van der Waals interaction force is available for two cylindrical shape nucleotides in the open literature. Here, the van der Waals force between two nucleotides are approximated using two spherical particles. By [1], [7], the van der Waals surface energy is given as:

$$
\begin{aligned}
\left(E_{v w}\right)_{i j}= & -\frac{H}{6}\left[\frac{d^{2}}{2\left(d+\delta_{i j}\right)^{2}}+\frac{d^{2}}{2 \delta_{i j}\left(2 d+\delta_{i j}\right)}\right. \\
& \left.+\ln \frac{\delta_{i j}\left(2 d+\delta_{i j}\right)}{\left(d+\delta_{i j}\right)^{2}}\right]
\end{aligned}
$$

where $H$ is the Hamaker constant. Here, $H=H_{1}-H_{2}$ is used to calculate the Hamaker constant [3]. $H_{1}$ is the Hamaker constant of the nucleotide and $H_{2}$ is the Hamaker constant of the propylene carbonate. The $d$ is the diameter of a nucleotide. The $\delta_{i j}$ is the shortest distance between two neighboring nucleotides. The van der Waals force can then be obtained as

$$
\begin{aligned}
\left(f_{v w}\right)_{i j} & =\frac{\partial\left(E_{v w}\right)_{i j}}{\partial \delta_{i j}} \\
& =\frac{H}{6}\left[\frac{d^{2}}{\left(d+\delta_{i j}\right)^{3}}+\frac{d^{2}\left(d+\delta_{i j}\right)}{\delta_{i j}^{2}\left(2 d+\delta_{i j}\right)^{2}}\right. \\
& \left.-\frac{2 d^{2}}{\delta_{i j}\left(2 d+\delta_{i j}\right)\left(d+\delta_{i j}\right)}\right]
\end{aligned}
$$

\section{Simulation Results}

By giving proper initial conditions, the contact dynamics of the above formulation can be simulated using Matlab. Units of physical variables and their values used in the simulation are given in Table I. The simulation was conducted for the two-dimensional space by assuming each nucleotide can freely interact with each other.

TABLE I

Physical Variables, Values and Description

\begin{tabular}{|c|c|c|}
\hline Var & Values & Descriptions \\
\hline$r$ & $0.5 \mathrm{~nm}$ & Radius of each DNA base \\
$h$ & $0.34 \mathrm{~nm}$ & Height of each DNA base \\
$m$ & $320 \mathrm{amu}$ & Average mass of a nucleotide \\
$\varepsilon_{0}$ & $8.85 \times 10^{-12} \mathrm{nF} / \mathrm{nm}$ & Permittivity of a vacuum \\
$\varepsilon_{r}$ & 88.0 & Relative dielectric constant \\
$k_{c}$ & $20 n N / n m$ & Linear contact stiffness \\
$c_{c}$ & $0.001 \mathrm{nN}-\mathrm{ns} / \mathrm{nm}$ & Linear contact damping coefficient \\
$c_{f}$ & $0.0001 \mathrm{nN}-\mathrm{ns} / \mathrm{nm}$ & translational viscous coefficient \\
$H$ & $6 \times 10^{-20} \mathrm{~J}$ & Hamaker constant \\
\hline
\end{tabular}

Fig. 3 shows positions of nine nucleotides from a simulation. The nine nucleotides are ejected at the same positions with different velocities in $x$ direction. Their offsets in $y$ direction vary from -0.5 to $0.5 \mathrm{~nm}$. It can be seen from the simulation that the nucleotides can all be attracted to the growing strand and built on top of each other. Within about 40 nano-second, the strand is stabilized. This time duration depends on initial positions and velocities of nucleotides. In reality, multi-nucleotides may contact each other, and only one base is built on top of the oligo nucleotide strand each time.
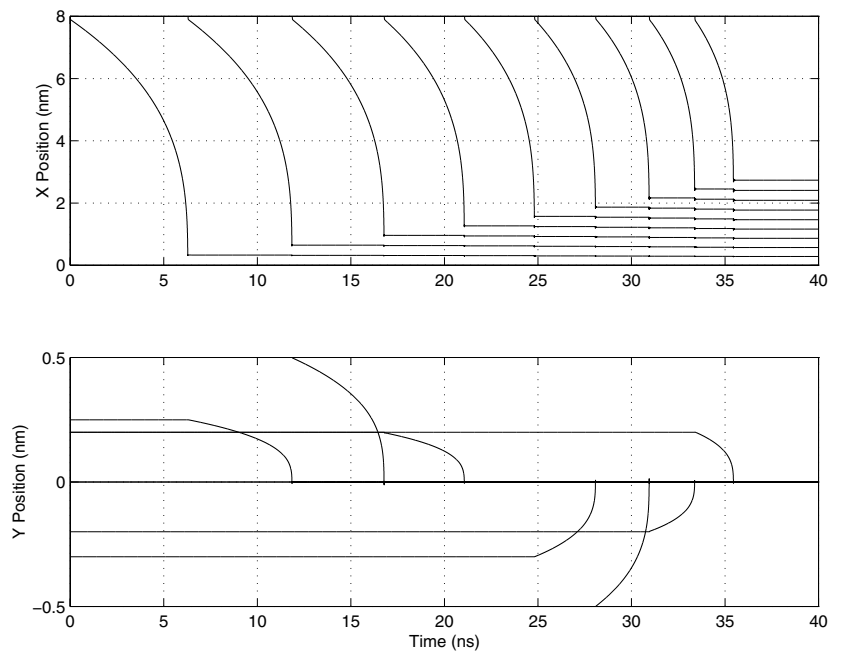

Fig. 3. Positions of Individual Nucleotides
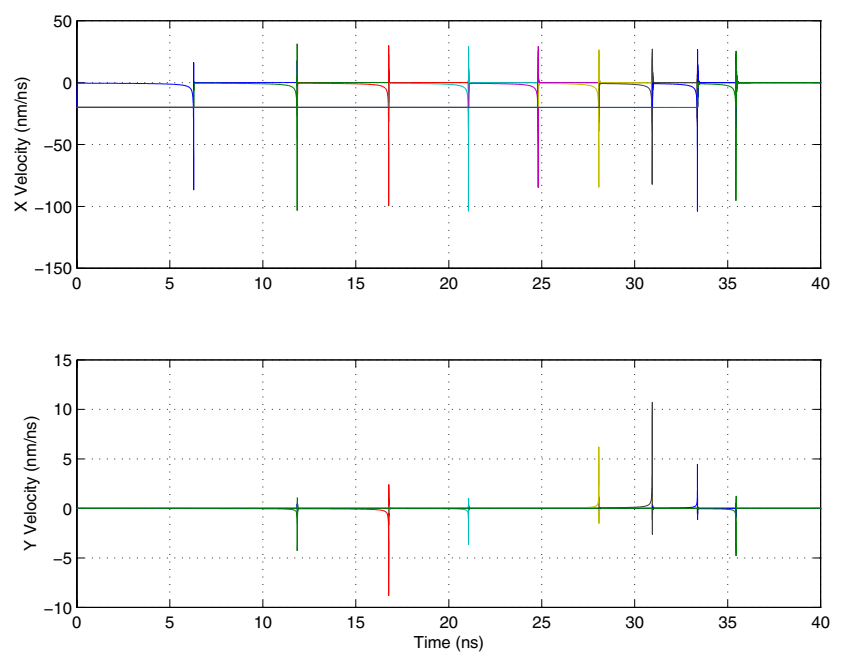

Fig. 4. Velocities of Individual Nucleotides

Fig. 4 shows velocities of the nine nucleotides. Fig. 8 shows the van der Waals forces of each nucleotide. Fig. 5, Fig. 6 and Fig. 8 show that the electrostatic force, the viscous force and the van der Waals force all contribute to the printing dynamics. The contact force as shown in Fig. 7 contributes significantly once the nucleotide get closer to each other. It should be noticed that there is no contact force in $y$-direction, since it is assumed that the surface is smooth and only the normal force exists in contact.

Some concerns may be raised for how to set the initial positions, velocities, and why the surface tension is not considered. In the simulation, we chose the initial state as the state when a droplet is close to contact the substrate surface. There are two reasons for this. First, the simulation can be shortened. Travel of the droplet before contacting to the surface is of less interest to us as opposed to contact motions. The initial position and velocity for simulation can be easily estimated based on travel distance from the print head. Second, the nucleotide dynamics can be considered 

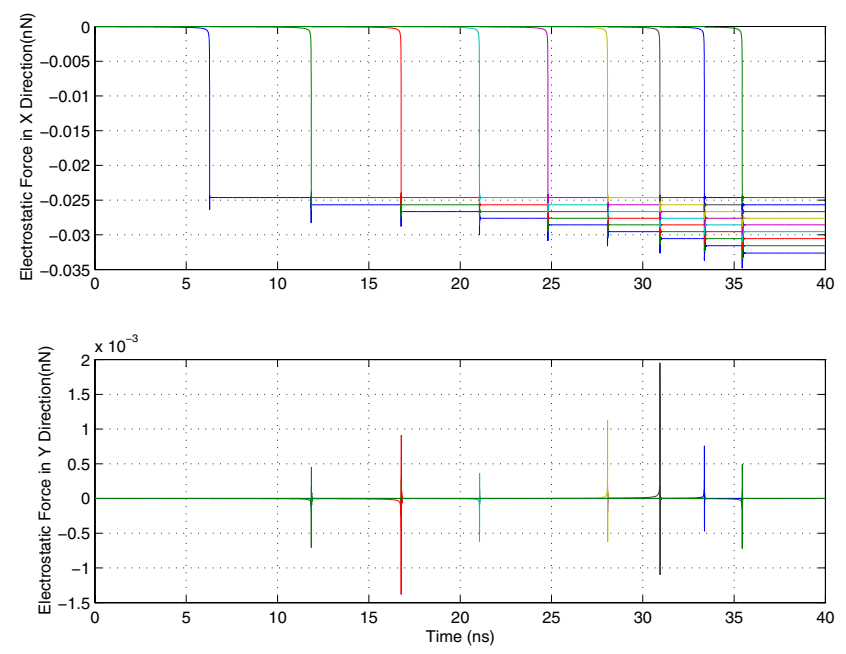

Fig. 5. Electrostatic Forces
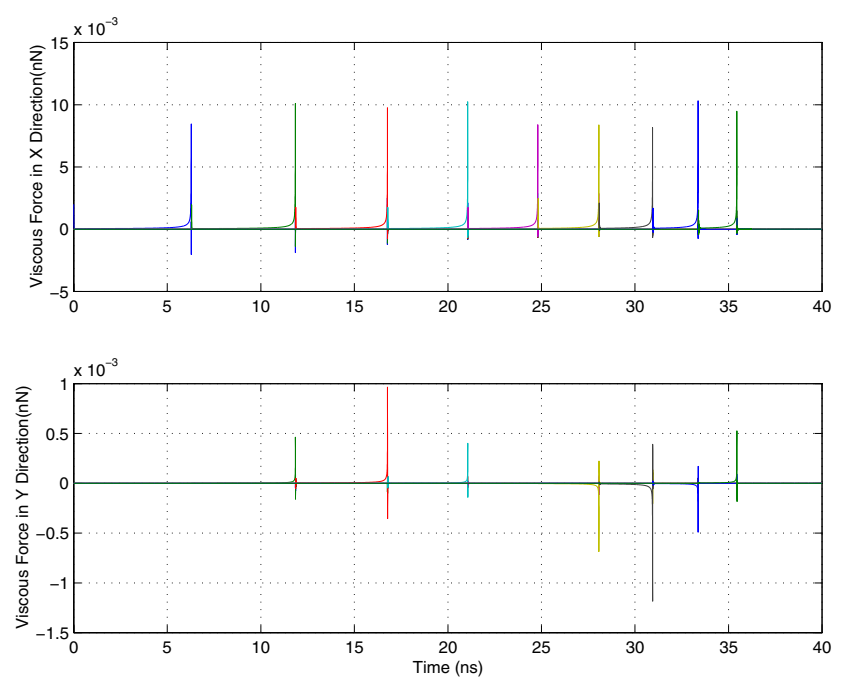

Fig. 6. Viscous Forces
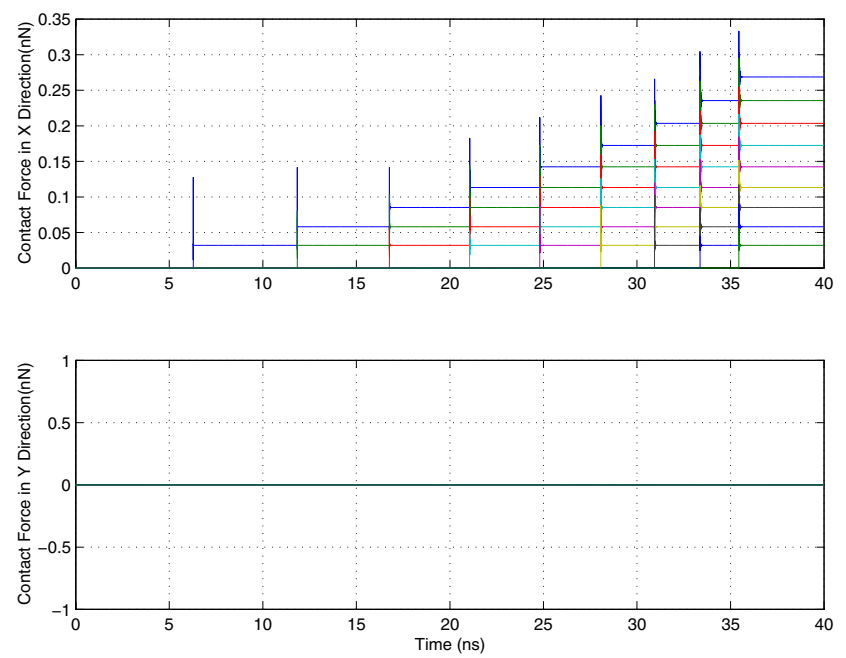

Fig. 7. Contact Forces
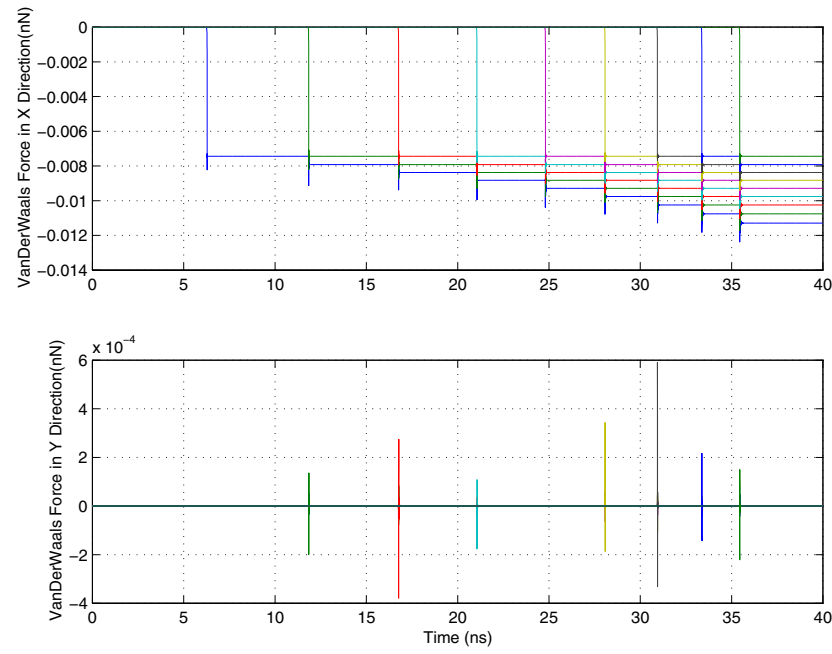

Fig. 8. Van Der Waals Forces

within the droplet (in a liquid media) by this simplification. No surface tension needs to be considered, which is an advantage for the dynamic modeling.

\section{A. Printing with different speeds}

To investigate how the nucleotide shooting velocity affects the inkjet printing process. Simulations were conducted for different initial velocities. It was noticed that the velocities did not affect the above dynamics much, if they are within certain range. It may be because the initial velocity is far less significant than the speeding up by the attraction forces. This conclusion is consistent with observation in practice. It is noticed in the microarray fabrication process that the shooting speed does not affect the fabrication results much as long as they are within certain range.

\section{B. Printing without electrostatic forces}

To find out how the chemically modified nucleotide end may contribute to the printing process, simulations were conducted without the electrostatic force. Fig. 9 shows positions of each nucleotide. The simulation confirms the observation in practice that the chemically modified nucleotide ends contributes significantly to the printing results. Without the modification, the nucleotide coupling process can not happen efficiently.

The Matlab simulation data was animated for visualization. Fig. 10 is a snapshot of the movie showing the printing process.

To summary, the above simulations can be used to answer some questions raised in practice. It is clear that the electrostatic and van der Waals forces both contribute to the nucleotide synthesis process. These forces are not significant and drop rapidly after increasing distance between the particles. To improve productivity of the nucleotide synthesis, it is important to have high density nucleotide solutions. In addition, the inkjet head firing speed and height do not affect the fabrication result much. Within certain range, the 

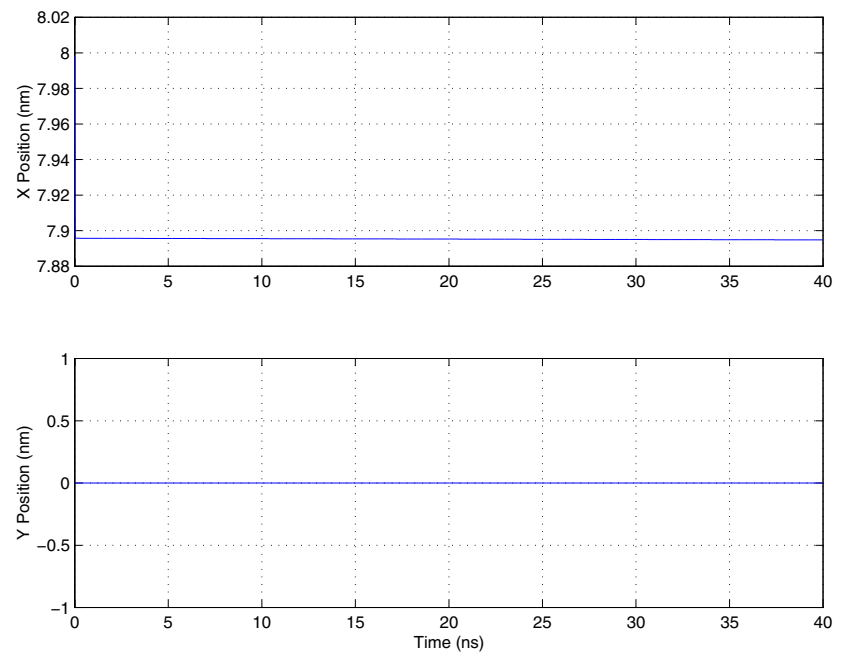

Fig. 9. Nucleotide Positions without electrostatic forces

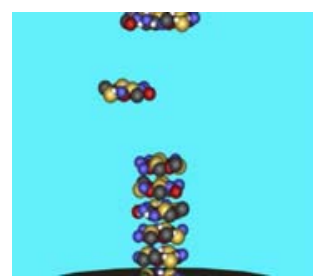

Fig. 10. Simulation Movie

fabrication result is not sensitive to either firing speed or height. Finally, the chemically modified nucleotide ends are critical for the nucleotide coupling process, since it is one of the forces drawing nucleotide close to the synthesized chains fast.

\section{Discussions AND CONCLUSIONS}

There are always trade-offs between complexity and applicability of dynamic modeling for a physical process. The above computational model is used to interpret sequential nucleotide contact dynamics behavior. In reality, a number of nucleotides may drop at the same time and multiple single stranded DNA may grow at same microarray spot. The dynamic modeling and analysis of such situation will be more complicated, but can be done by extending the above modeling approach. In addition, single stranded DNA molecules are not a rigid, static structure. In reality, it is under constant thermal fluctuations, which results in local twisting, stretching, and bending. Above thermal fluctuations are omitted in this formulation, since the nucleotide contact is considered to be the major contribution to the printing dynamics.

In summary, it is interesting to report that, by introducing molecular interaction forces and using classical contact mechanics, a computational model can be built and the model can reasonably well explain dynamics of the microarray spotting process.

\section{REFERENCES}

[1] F. Arai, D. Ando, and T. Fukuda. Micro-manipulation based on micro-physics: strategy based on attractive force reduction and stress measurement, Proceedings of the 1995 International Conference on Robotics and Automation, Pittsburgh, PA, pp. 236-241, 1995.

[2] W. D. Callister. Materials science and engineering: an introduction, 4th edition, John Wiley \& Sons, 1997.

[3] J. Israelachvili. Intermolecular and surface forces, 2nd edition, London UK. Academic, 1992.

[4] C. Maier, S. Wiesche and E. P. Hofer. Impact of microdrops on solid surfaces for DNA-synthesis, Proceedings of the 2000 International Conference on Modeling and Simulation of Microsystems, pp. 586589, 2000.

[5] T. Schlick, D. A. Beard, J. Huang, et al. Computational changes in simulating large DNA over long times, IEEE Journal of Computig in Science \& Engineering, pp. 38-51, November/December 2000.

[6] M. Sitti, and H. Hashimoto. Controlled pushing of nanoparticles: modeling and experiments, IEEE/ASME Trans. on Mechatronics, vol. 5, no. 2, pp. 199-211, 1999.

[7] R. Tadmor. The London-van der Waals interaction energy between objects of various geometries, J. Physics: Condens. Matter, vol. 13, L195-202, 2001.

[8] Mingjun Zhang, K. Griswold, W. Fisher and T. J. Tarn. DNA Microarray Manufacturing Factory Automation, Proceedings of 2003 IEEE International Conference on Robotics and Automation, pp. 2895-2900, Sept. 2003, Taipei, Taiwan. 